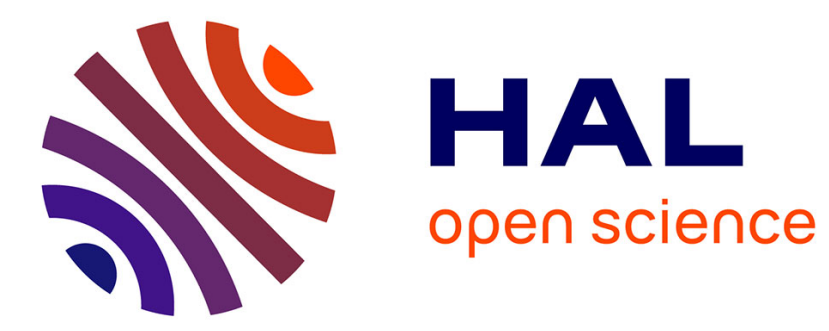

\title{
Robust sampled-data stabilization of linear systems - An input delay approach
}

\author{
Emilia Fridman, Alexandre Seuret, Jean-Pierre Richard
}

\section{To cite this version:}

Emilia Fridman, Alexandre Seuret, Jean-Pierre Richard. Robust sampled-data stabilization of linear systems - An input delay approach. Automatica, 2004. inria-00131031

\section{HAL Id: inria-00131031 \\ https://inria.hal.science/inria-00131031}

Submitted on 28 Nov 2007

HAL is a multi-disciplinary open access archive for the deposit and dissemination of scientific research documents, whether they are published or not. The documents may come from teaching and research institutions in France or abroad, or from public or private research centers.
L'archive ouverte pluridisciplinaire HAL, est destinée au dépôt et à la diffusion de documents scientifiques de niveau recherche, publiés ou non, émanant des établissements d'enseignement et de recherche français ou étrangers, des laboratoires publics ou privés. 


\title{
Robust Sampled-Data Stabilization of Linear Systems: An Input Delay Approach
}

\author{
Emilia Fridman*, $\quad$ Alexandre Seuret ${ }^{\dagger}, \quad$ Jean-Pierre Richard ${ }^{\dagger}$ \\ * Department of Electrical Engineering-Systems \\ Tel-Aviv University, Tel-Aviv 69978, Israel. \\ e-mail: emilia@eng.tau.ac.il \\ † LAIL UMR 8021 Ecole Centrale de Lille, 59651 Villeneuve d'ascq cedex \\ e-mail: Seuret.Alexandre; jean-pierre.richard@ec-lille.fr
}

\begin{abstract}
A new approach to robust sampled-data control is introduced. The system is modelled as a continuous-time one, where the control input has a piecewise-continuous delay. Sufficient linear matrix inequalities (LMIs) conditions for sampled-data state-feedback stabilization of such systems are derived via descriptor approach to time-delay systems. The only restriction on the sampling is that the distance between the sequel sampling times is not greater than some prechosen $h>0$ for which the LMIs are feasible. For $h \rightarrow 0$ the conditions coincide with the necessary and sufficient conditions for continuous-time state-feedback stabilization. Our approach is applied to two problems: sampled-data stabilization of systems with polytopic type uncertainties and to regional stabilization by sampled-data saturated state-feedback.
\end{abstract}

Keywords: Sampled-data control, stabilization, input delay, LMI, time-varying delay

\section{Introduction}

Modelling of continuous-time systems with digital control in the form of continuous-time systems with delayed control input was introduced by Mikheev, Sobolev \& Fridman (1988), Astrom \& Wittenmark (1989) and further developed by Fridman (1992). The digital control law may be represented as delayed control as follows:

$$
u(t)=u_{d}\left(t_{k}\right)=u_{d}\left(t-\left(t-t_{k}\right)\right)=u_{d}(t-\tau(t)), \quad t_{k} \leq t<t_{k+1}, \tau(t)=t-t_{k},
$$

where $u_{d}$ is a discrete-time control signal and the time-varying delay $\tau(t)=t-t_{k}$ is piecewise-linear with derivative $\dot{\tau}(t)=1$ for $t \neq t_{k}$. Moreover, $\tau \leq t_{k+1}-t_{k}$. Based on such a model, for small enough sampling intervals $t_{k+1}-t_{k}$ asymptotic approximations of the trajectory (Mikheev et al, 1988) and of the optimal solution to the sampled-data LQ finite horizon problem (Fridman, 1992) were constructed. 
Since the middle of 90 's years of the last century different LMI conditions for robust stability of linear systems with uncertain, but bounded constant delay with a given upper bound have been derived (see e.g. Li \& De Souza, 1997;Niculescu, 2001). For systems with time-varying delays such conditions were obtained via Lyapunov-Krasovskii functionals in the case where the derivative of the delay is less than one (see e.g. Niculescu, de Souza, Dugard \& Dion, 1998). The stability issue in the cases of time-varying delay without any restrictions on the derivative of the delay has been treated mainly via Lyapunov-Razumikhin functions, which usually lead to conservative results (see e.g. Hale \& Lunel, 1993; Cao, Sun \& Cheng, 1998; Kolmanovskii \& Myshkis, 2000; Niculescu, 2001).Only recently for the first time this case was treated by Lyapunov-Krasovskii technique (Fridman \& Shaked, 2002). This became possible due to a new descriptor model representation of the delay system introduced by Fridman (2001).

Two main approaches have been used to the sampled-data robust stabilization problem (see e.g. Dullerud \& Glover, 1993; Sivashankar \& Khargonekar, 1993; Basar \& Bernard, 1995; Oishi, 1997). The first one is based on the lifting technique (Bamieh \& Pearson, 1992; Yamamoto, 1990) in which the problem is transformed to equivalent finite-dimensional discrete problem. However, this approach does not work in the cases with uncertain sampling times or uncertain system matrices.

The second approach is based on the representation of the system in the form of hybrid discrete/continuous model. Application of this approach to linear systems leads to necessary and sufficient conditions for stability and $L_{2}$-gain analysis in the form of differential equations (or inequalities) with jumps (see e.g. Sivashankar \& Khargonekar, 1994). The latter approach has been applied recently to sampled-data stabilization of linear uncertain systems for the case of equidistant sampling (Hu, Cao \& Shao, 2002; Hu, Cao, Cheng \& Shao,2002; Hu, Lam, Cao \& Shao, 2003). To overcome difficulties of solving differential inequalities with jumps, a piecewise-linear in time Lyapunov function has been suggested. As a result in (Hu, Cao \& Shao, 2002; Hu, Cao, Cheng \& Shao,2002) a countable sequence of matrix inequalities has been derived, which has been proposed to solve by iterative method. The feasibility of these matrix inequalities is not guaranteed even for small sampling periods. In the most recent work (Hu et al., 2003) LMIs have been derived (see Corollary 2) which do not depend on the sampling interval and thus are very conservative.

In the present paper we suggest a new approach to the robust sampled-data stabilization. We find a solution by solving the problem for a continuous-time system with uncertain but bounded (by the maximum sampling interval) time-varying delay in the control input. We verify that the LMI sufficient conditions for stability of (Fridman \& Shaked, 2002) are valid also in the case of piecewise-continuous delay and derive LMIs for the feedback gain. The conditions which we obtain are robust with respect to different samplings with the only requirement that the maximum sampling interval is not greater than $h$. Moreover, the feasibility of the LMIs is guaranteed for small $h$ if the corresponding continuous-time controller stabilizes the system. As a by-product we show that for $h \rightarrow 0$ the conditions coincide with the necessary and sufficient conditions for the continuous-time 
stabilization. Such convergence in $H_{2}$ framework and related results were proved by Mikheev et al (1988); Chen \& Francis (1991); Fridman (1992); Osborn \& Bernshtein (1995); Trentelman \& Stoorvogel (1995), Oishi (1997).

For the first time the new approach allows to develop different robust control methods for the case of sampled-data control. The LMIs are affine in the system matrices and thus for the systems with polytopic type uncertainty the stabilization conditions readily follow. We consider the regional stabilization by sampled-data saturated state-feedback, where we give an estimate on the domain of attraction. For continuous-time stabilization of state-delayed systems by saturated-feedback see e.g. Dambrine, Richard \& Borne (1994); Tarbouriech \& Gomes da Silva , 2000; Cao, Lin \& Hu, 2002; Fridman, Pila \& Shaked, 2003.

Notation: Throughout the paper the superscript ' $T$ ' stands for matrix transposition, $\mathcal{R}^{n}$ denotes the $n$ dimensional Euclidean space with vector norm $|\cdot|, \mathcal{R}^{n \times m}$ is the set of all $n \times m$ real matrices, and the notation $P>0$, for $P \in \mathcal{R}^{n \times n}$ means that $P$ is symmetric and positive definite. Given $\bar{u}=\left[\bar{u}_{1}, \ldots, \bar{u}_{m}\right]^{T}, 0<\bar{u}_{i}, i=1, \ldots, m$, for any $u=\left[u_{1}, \ldots, u_{m}\right]^{T}$ we $\operatorname{denote}$ by $\operatorname{sat}(u, \bar{u})$ the vector with coordinates $\operatorname{sign}\left(u_{i}\right) \min \left(\left|u_{i}\right|, \bar{u}_{i}\right)$. By stability of the system we understand the asymptotic stability of it.

\section{Sampled-data stabilization of uncertain systems}

\subsection{Problem Formulation}

Consider the system

$$
\dot{x}(t)=A x(t)+B u(t),
$$

where $x(t) \in \mathcal{R}^{n}$ is the state vector, $u(t) \in \mathcal{R}^{m}$ is the control input. We are looking for a piecewiseconstant control law of the form $u(t)=u_{d}\left(t_{k}\right), t_{k} \leq t<t_{k+1}$, where $u_{d}$ is a discrete-time control signal and $0=t_{0}<t_{1}<\ldots<t_{k}<\ldots$ are the sampling instants. Our objective is to find a state-feedback controller given by

$$
u(t)=K x\left(t_{k}\right), t_{k} \leq t<t_{k+1}
$$

which stabilizes the system.

We represent a piecewise-constant control law as a continuous-time control with a time-varying piecewise-continuous (continuous from the right) delay $\tau(t)=t-t_{k}$ as given in (1). We will thus look

for a state-feedback controller of the form: $u(t)=K x(t-\tau(t))$. Substituting the latter controller into (2), we obtain the following closed-loop system:

$$
\dot{x}(t)=A x(t)+B K x(t-\tau(t)), \quad \tau(t)=t-t_{k}, t_{k} \leq t<t_{k+1} .
$$

We assume that 
A1 $t_{k+1}-t_{k} \leq h \forall k \geq 0$.

From A1 it follows that $\tau(t) \leq h$ since $\tau(t) \leq t_{k+1}-t_{k}$. We will further consider (4) as the system with uncertain and bounded delay.

\subsection{Stability of the closed-loop system}

Similarly to Fridman \& Shaked (2002), where the continuous delay was considered, we obtain for the case of piecewise-continuous delay the following result:

Lemma 2.1 Given a gain matrix $K$, the system (4) is stable for all the samplings satisfying A1, if there exist $n \times n$ matrices $0<P_{1}, P_{2}, P_{3}, Z_{1}, Z_{2}, Z_{3}$ and $R>0$ that satisfy the following LMIs:

$$
\Psi_{1}<0, \quad \text { and } \quad\left[\begin{array}{cc}
R & {\left[0 K^{T} B^{T}\right] P} \\
* & Z
\end{array}\right] \geq 0,
$$

where

$$
\begin{aligned}
& P=\left[\begin{array}{cc}
P_{1} & 0 \\
P_{2} & P_{3}
\end{array}\right], \quad Z=\left[\begin{array}{cc}
Z_{1} & Z_{2} \\
* & Z_{3}
\end{array}\right], \quad \Psi_{1}=\Psi_{0}+h Z+\left[\begin{array}{cc}
0 & 0 \\
0 & h R
\end{array}\right], \\
& \Psi_{0}=P^{T}\left[\begin{array}{cc}
0 & I \\
A+B K & -I
\end{array}\right]+\left[\begin{array}{cc}
0 & I \\
A+B K & -I
\end{array}\right]^{T} P .
\end{aligned}
$$

Proof is based on the following descriptor representation of (4) (Fridman, 2001):

$$
\dot{x}(t)=y(t), \quad 0=-y(t)+A x(t)+B K x(t-\tau(t)),
$$

or equivalently

$$
\dot{x}(t)=y(t), \quad 0= \begin{cases}-y(t)+A x(t)+B K x(t-\tau(t)), & \text { if } t \in[0, h), \\ -y(t)+(A+B K) x(t)-B K \int_{t-\tau(t)}^{t} y(s) d s, & \text { if } t \geq h,\end{cases}
$$

which is valid in the case of piecewise-continuous delay $\tau(t)$ for $t \geq 0$. Given a matrix $K$ and initial condition $x(t)=\phi(t)(t \in[-h, 0])$, where $\phi$ is a continuous function, $x(t)$ satisfies (4) for $t \geq 0$ iff it satisfies (6) (or equivalently (7)). Note that the descriptor system (6) has no impulsive solutions since in (6b) $y(t)$ is multiplied by the nonsingular matrix $I$ (Fridman, 2002).

We apply the Lyapunov-Krasovskii functional of the form

$$
V(t)=V_{1}+V_{2}, \quad V_{1}=\bar{x}^{T}(t) E P \bar{x}(t), V_{2}=\int_{-h}^{0} \int_{t+\theta}^{t} y^{T}(s) R y(s) d s d \theta
$$

where

$$
\bar{x}(t)=\operatorname{col}\{x(t), y(t)\}, \quad E=\left[\begin{array}{rr}
I_{n} & 0 \\
0 & 0
\end{array}\right], \quad P=\left[\begin{array}{rr}
P_{1} & 0 \\
P_{2} & P_{3}
\end{array}\right], \quad P_{1}=P_{1}^{T}>0,
$$

which satisfies the following inequalities

$$
a|x(t)|^{2} \leq V(t) \leq b \sup _{s \in[-h, 0]}|\bar{x}(t+s)|^{2}, \quad a>0, b>0 .
$$


Differentiating $V(t)$ along the trajectories of (7) for $t \geq h$ we find (see Fridman \& Shaked, 2002) that

$$
\dot{V}(t)<\bar{x}^{T}(t) \Psi_{1} \bar{x}(t)<-c|x(t)|^{2}, \quad c>0, t \geq h,
$$

provided that $(5 \mathrm{a}, \mathrm{b})$ hold. Integrating $(11)$ we have

$$
V(t)-V(h) \leq-c \int_{h}^{t}|x(s)|^{2} d s
$$

and, hence, (10) yields

$$
|x(t)|^{2} \leq V(t) / a \leq V(h) / a \leq b / a \sup _{s \in[-h, 0]}|\bar{x}(h+s)|^{2} .
$$

Since $\sup _{s \in[-h, 0]}|x(h+s)| \leq c_{1} \sup _{s \in[-h, 0]}|\phi(s)|, c_{1}>0$ (cf. p. 168 of Hale \& Lunel, 1993) and thus $\dot{x}$, defined by the right-hand side of (4), satisfies $\sup _{s \in[-h, 0]}|\dot{x}(h+s)| \leq c_{2} \sup _{s \in[-h, 0]}|\phi(s)|, c_{2}>0$, we obtain that

$$
|x(t)|^{2} \leq V(h) / a \leq c_{3} \sup _{s \in[-h, 0]}|\phi(s)|, c_{3}>0 .
$$

Hence, (4) is stable (i.e. $x(t)$ is bounded and small for small $\phi$ ).

To prove asymptotic stability we note that $x(t)$ is uniformly continuous on $[0, \infty)$ (since $\dot{x}(t)$ defined by the right-hand side of (4) is uniformly bounded). Moreover, (12) yields that $|x(t)|^{2}$ is integrable on $[h, \infty)$. Then, by Barbalat's lemma, $x(t) \rightarrow 0$ for $t \rightarrow \infty$.

Applying now the continuous state-feedback $u(t)=K x(t)$ to $(2)$ we obtain the system

$$
\dot{x}(t)=(A+B K) x(t) .
$$

It is clear that the stability of (13) is equivalent to the stability of its equivalent descriptor form

$$
\dot{x}(t)=y(t), \quad 0=-y(t)+(A+B K) x(t),
$$

which coincides with (6) for $h=0$. It is well-known (Takaba, Morihira \& Katayama, 1995) that the stability of the latter system is equivalent to the condition $\Psi_{0}<0$.

If there exists $P$ of the form $(9 \mathrm{c}, \mathrm{d})$ which satisfies $\Psi_{0}<0$, then for small enough $h>0$ LMIs of Lemma 2.1 are feasible (take e.g. $Z=I_{2 n}$ and $R=\left[\begin{array}{lll}0 & K^{T} & B^{T}\end{array} P^{T} P\left[\begin{array}{ll}0 & K^{T}\end{array} B^{T}\right]^{T}\right.$ ). We, therefore, obtain the following result:

Corollary 2.2 If the continuous-time state-feedback $u(t)=K x(t)$ stabilizes the linear system (2), then the sampled-data state-feedback (3) with the same gain $K$ stabilizes (2) for all small enough $h$.

Remark 1 In the case where the matrices of the system are not exactly known, we denote $\Omega=$ $\left[\begin{array}{ll}A & B\end{array}\right]$ and assume that $\Omega \in \mathcal{C} o\left\{\Omega_{j}, j=1, \ldots N\right\}$, namely,

$$
\Omega=\sum_{j=1}^{N} f_{j} \Omega_{j} \quad \text { for some } \quad 0 \leq f_{j} \leq 1, \sum_{j=1}^{N} f_{j}=1
$$


where the $N$ vertices of the polytope are described by $\Omega_{j}=\left[\begin{array}{ll}A^{(j)} & B^{(j)}\end{array}\right]$. In order to guarantee the stability of (2) over the entire polytope one can use the result of Lemma 2.1 by applying the same matrices $P_{2}$ and $P_{3}$ for all the points in the polytope and solving (5a,b) for the $N$ vertices only.

\subsection{Sampled-Data Stabilization}

LMIs of Lemma 2.1 are bilinear in $P$ and $K$. In order to obtain LMIs we use $P^{-1}$. It is obvious from the requirement of $0<P_{1}$, and the fact that in $(5)-\left(P_{3}+P_{3}^{T}\right)$ must be negative definite, that $P$ is nonsingular. Define

$$
P^{-1}=Q=\left[\begin{array}{cc}
Q_{1} & 0 \\
Q_{2} & Q_{3}
\end{array}\right] \text { and } \Delta=\operatorname{diag}\{Q, I\}
$$

Applying Schur formula to the term $h R$ in (5a), we multiply $(5 \mathrm{a}, \mathrm{b})$ by $\Delta^{T}$ and $\Delta$, on the left and on the right, respectively. Denoting $\bar{R}=R^{-1}$ and $\bar{Z}=Q^{T} Z Q$ we obtain, similarly to (Fridman and Shaked 2002), the following

Lemma 2.3 The control law of (3) stabilizes (2) for all the samplings with the maximum sampling interval not greater than $h$ and for all the system parameters that reside in the uncertainty polytope $\Omega$, if there exist: $Q_{1}>0, Q_{2}^{(j)}, Q_{3}^{(j)}, \bar{R}, \bar{Z}_{1}^{(j)}, \bar{Z}_{2}^{(j)}, \bar{Z}_{3}^{(j)} \in \mathcal{R}^{n \times n}, \bar{Y} \in \mathcal{R}^{q \times n}$ that satisfy the following nonlinear matrix inequalities:

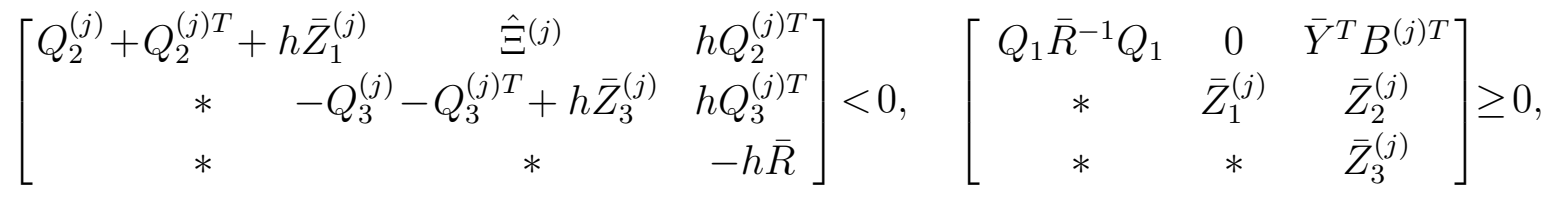

where

$$
\hat{\Xi}^{(j)}=Q_{3}^{(j)}-Q_{2}^{(j) T}+Q_{1} A^{(j) T}+h \bar{Z}_{2}^{(j)}+\bar{Y}^{T} B^{(j) T}, \quad j=1,2, \ldots, N .
$$

The state-feedback gain is then given by $K=\bar{Y} Q_{1}^{-1}$.

For solving (17) there exist two methods. The first uses the assumption

$$
\bar{R}=\varepsilon Q_{1}, \quad \varepsilon>0
$$

and thus leads to $2 N$ LMIs with tuning parameter $\varepsilon$ :

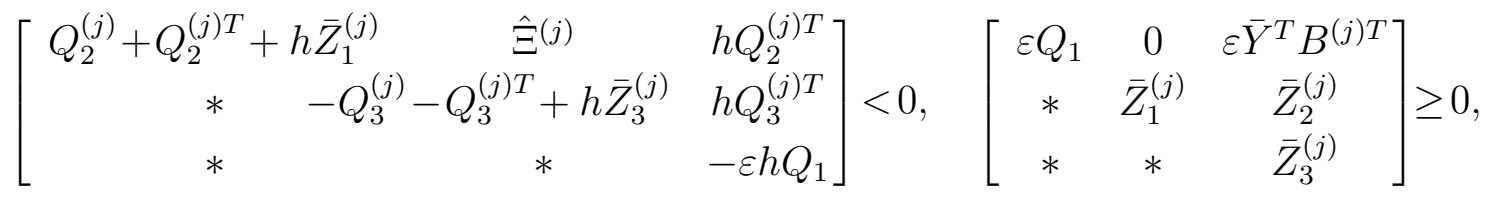

where $\hat{\Xi}^{(j)}$ and $j$ are given by (18). 
Similarly to Corollary 2.2 we can show that if the system (2) is quadratically stabilizable by a continuous-time state-feedback $u(t)=K x(t)$, then for all small enough $h$ the latter LMIs are feasible and the sampled-data state-feedback with the same gain stabilizes the system.

The second method for solving (17) is based on the iterative algorithm developed recently by Gao and Wang (2003). This method is preferable in the cases of comparatively large $h$, since it leads to less conservative results. However it may take more computer time due to iterative process. In the sequel we shall adopt the first method for solving (17).

Example 1 We consider (2) with the following matrices:

$$
A=\left[\begin{array}{cc}
1 & 0.5 \\
g_{1} & -1
\end{array}\right], \quad B=\left[\begin{array}{c}
1+g_{2} \\
-1
\end{array}\right],
$$

where $\left|g_{1}\right| \leq 0.1,\left|g_{2}\right| \leq 0.3$. It is verified by using (20) that for all uncertaintires the system is stabilizable by a sampled-data state-feedback with the maximum sampling interval $h \leq 0.35$. Thus, for $h=0.35$ the resulting $K=[-2.6884-0.6649]$ (with $\varepsilon=0.7$ ). Simulation results (for uniform samplings with the $g_{1}=0.1$ sint, $g_{2}=0.3$ cost and sampling interval less than ?) show that the closed-loop system is stable. For $h=$ and $g_{1}, g_{2}$ as above the system becomes unstable.

\section{Stabilization by Saturated Sampled-Data Controller}

\subsection{Problem formulation}

Consider the system (2) with the sampled-data control law (3) which is subject to the following amplitude constraints

$$
\left|u_{i}(t)\right| \leq \bar{u}_{i}, \quad 0<\bar{u}_{i}, i=1, \ldots, m
$$

We represent the state-feedback in the delayed form $u(t)=\operatorname{sat}(K x(t-\tau(t)), \bar{u}), \tau(t)=t-t_{k}, t_{k} \leq$ $t<t_{k+1}$. Applying the latter control law the closed-loop system obtained is

$$
\dot{x}(t)=A x(t)+B \operatorname{sat}(K x(t-\tau(t)), \bar{u}), \tau(t)=t-t_{k}, t_{k} \leq t<t_{k+1} .
$$

Though the closed-loop system (22) has a delay, in the case of sampled-data control the initial condition is defined in the point $t=0$ and not in the segment $[-h, 0]$. Denote by $x(t, x(0))$ the state trajectory of $(22)$ with the initial condition $x(0) \in R^{n}$. Then the domain of attraction of the origin of the closed-loop system (22) is the set

$$
\mathcal{A}=\left\{x(0) \in R^{n}: \lim _{t \rightarrow \infty} x(t, x(0))=0\right\} .
$$

We seek conditions for the existence of a gain matrix $K$ which leads to a stable closed-loop. Having met these conditions, a simple procedure for finding the gain $K$ should be presented. Moreover, we obtain an estimate $\mathcal{X}_{\beta} \subset \mathcal{A}$ on the domain of attraction, where

$$
\mathcal{X}_{\beta}=\left\{x(0) \in R^{n}: x^{T}(0) P_{1} x(0) \leq \beta^{-1}\right\}
$$


and where $\beta>0$ is a scalar and $P_{1}>0$ is an $n \times n$ matrix.

Reducing the original problem to the problem with input delay, we solve it by modifying derivations of (Fridman et al, 2003), where the case of state delay was considered.

\subsection{A linear system representation with polytopic type uncertainty}

Since the initial condition for (22) is defined in the point $t=0$, for the estimation of $\mathcal{A}$ we can restrict ourself to the following initial functions $\phi(s), s \in[-h, 0]$ :

$$
\phi(0)=x(0), \phi(s)=0, s \in[-h, 0) .
$$

Denoting the i-th row by $k_{i}$, we define the polyhedron

$$
\mathcal{L}(K, \bar{u})=\left\{x \in \mathcal{R}^{n}:\left|k_{i} x\right| \leq \bar{u}_{i}, i=1, \ldots, m\right\} .
$$

If the control and the disturbance are such that $x \in \mathcal{L}(K, \bar{u})$ then the system (22) admits the linear representation. Following (Cao et al, 2002), we denote the set of all diagonal matrices in $\mathcal{R}^{m \times m}$ with diagonal elements that are either 1 or 0 by $\Upsilon$, then there are $2^{m}$ elements $D_{i}$ in $\Upsilon$, and for every $i=1, \ldots, 2^{m} D_{i}^{-} \triangleq I_{m}-D_{i}$ is also an element in $\Upsilon$.

Lemma 3.1 (Cao et al, 2002) Given $K$ and $H$ in $\mathcal{R}^{m \times n}$. Then

$$
\operatorname{sat}(K x(t), \bar{u}) \in \mathcal{C} o\left\{D_{i} K x+D_{i}^{-} H x, i=1, \ldots, 2^{m}\right\}
$$

for all $x \in \mathcal{R}^{n}$ that satisfy $\left|h_{i} x\right| \leq \bar{u}_{i}, i=1, \ldots, 2^{m}$.

The following is obtained from Lemma 3.1.

Lemma 3.2 Given $\beta>0$, assume that there exists $H$ in $\mathcal{R}^{m \times n}$ such that $\left|h_{i} x\right| \leq \bar{u}_{i}$ for all $x(t) \in$ $\mathcal{X}_{\beta}$. Then for $x(t) \in \mathcal{X}_{\beta}$ the system (22) admits the following representation.

$$
\dot{x}(t)=A x(t)+\sum_{j=1}^{2^{m}} \lambda_{j}(t) A_{j} x(t-\tau(t))
$$

where

$$
A_{j}=B\left(D_{j} K+D_{j}^{-} H\right) \quad j=1, \ldots, 2^{m}, \sum_{j=1}^{2^{m}} \lambda_{j}(t)=1,0 \leq \lambda_{j}(t), \forall 0<t,
$$

We denote

$$
\Omega_{\alpha}=\sum_{j=1}^{2^{m}} \lambda_{j} \Omega_{j} \quad \text { for all } \quad 0 \leq \lambda_{j} \leq 1, \sum_{j=1}^{2^{m}} \lambda_{j}=1
$$

where the vertices of the polytope are described by $\Omega_{j}=\left[A_{j}\right], \quad j=1, \ldots, 2^{m}$. The problem becomes one of finding $\mathcal{X}_{\beta}$ and a corresponding $H$ such that $\left|h_{i} x\right| \leq \bar{u}_{i}, i=1, \ldots 2^{m}$ for all $x \in \mathcal{X}_{\beta}$ and that the state of the system

$$
\dot{x}(t)=A x(t)+A_{j} x(t-\tau(t)), \tau(t)=t-t_{k}, t_{k} \leq t<t_{k+1},
$$

remains in $\mathcal{X}_{\beta}$. 


\subsection{Regional stabilization}

By using the first method for solving the stabilization matrix inequalities (with tuning parameter $\varepsilon)$, we obtain the following result:

Theorem 3.3 Consider the system (2) with the constrained sampled-data control law (3). The closed-loop system (22) is stable with $\mathcal{X}_{\beta}$ inside the domain of attraction for all the samplings with the maximum sampling interval not greater than $h$, if there exist $0<Q_{1}, Q_{2}^{(j)}, Q_{3}^{(j)}, \bar{Z}_{1}^{(j)}, \bar{Z}_{2}^{(j)}, \bar{Z}_{3}^{(j)} \in$ $\mathcal{R}^{n \times n}, \bar{Y}, G \in \mathcal{R}^{m \times n}, \varepsilon>0$ and $\beta>0$ that satisfy $L M I$ (20a) for $j=1, \ldots, 2^{m}$, where

$$
\Sigma_{j}=Q_{3}^{(j)}-Q_{2}^{T(j)}+Q_{1} A^{T}+\left(\bar{Y}^{T} D_{j}+G^{T} D_{j}^{-}\right) B^{T}+h \bar{Z}_{2}^{(j)}, B^{(j)}=B
$$

and

$$
\left[\begin{array}{ccc}
\varepsilon Q_{1} & 0 & \varepsilon\left(\bar{Y}^{T} D_{j}+G^{T} D_{j}^{-}\right) B^{T} \\
* & \bar{Z}_{1}^{(j)} & \bar{Z}_{2}^{(j)} \\
* & * & \bar{Z}_{3}^{(j)}
\end{array}\right] \geq 0, j=1, \ldots, 2^{m}, \quad\left[\begin{array}{cc}
\beta & g_{i} \\
* & \bar{u}_{i}^{2} Q_{1}
\end{array}\right] \geq 0, i=1, \ldots, m .
$$

The feedback gain matrix which stabilizes the system is given by $K=\bar{Y} Q_{1}^{-1}$.

Proof: For $V$ given by (8) conditions are sought to ensure that $\dot{V}<0$ for any $x(t) \in \mathcal{X}_{\beta}$. As in (Fridman et al., 2003), the inequalities (30b) guarantee that $\left|h_{i} x\right| \leq \bar{u}_{i}, \forall x \in \mathcal{X}_{\beta}, i=1, \ldots, m$, where $g_{i} \triangleq h_{i} Q_{1}, i=1, \ldots, m$ and $Q_{1} \triangleq P_{1}^{-1}$, and the polytopic system representation of (28) is thus valid. Moreover, (20a,b) guarantee that $\dot{V}<0$.

From $\dot{V}<0$ it follows that $V(t)<V(0)$ and therefore for the initial conditions of the form $(24)$

$$
x^{T}(t) P_{1} x(t) \leq V(t)<V(0)=x^{T}(0) P_{1} x(0) \leq \beta^{-1} .
$$

Then for all initial values $x(0) \in \mathcal{X}_{\beta}$, the trajectories of $x(t)$ remain within $\mathcal{X}_{\beta}$, and the polytopic system representation (28) is valid. Hence $x(t)$ is a trajectory of the linear system (28) and $\dot{V}<0$ along the trajectories of the latter system which implies that $\lim _{t \rightarrow \infty} x(t)=0$.

Example 2. We consider (2) with the following matrices ( (Cao et al, 2002), where $h=0$ ):

$$
A=\left[\begin{array}{cc}
1.1 & -0.6 \\
.5 & -1
\end{array}\right], \quad B_{1}=\left[\begin{array}{l}
1 \\
1
\end{array}\right]
$$

and where $\bar{u}=5$. Applying Theorem 3.3 a stabilizing gain was obtained for all samplings with the maximum sampling interval $h \leq 0.75$. In order to 'enlarge' the volume of the ellipse we minimized the value of $\beta$ (to improve the result we also added the inequality $Q_{1}>\alpha I$ and chose such $\alpha>0$ that enlarged the resulting ellipse). The ellipse volume increases when $h$ decreases (see Figure 1). For, say, $h=0.75$ we obtain $K=[-1.69640 .5231]$ (with $\varepsilon=0.325, \beta=0.1261, P_{1}=$

$\left[\begin{array}{cc}0.9132 & -0.2816 \\ -0.2816 & 0.0868\end{array}\right], \alpha=1$ ) and we show (see Figure 2) that a trajectory starting on the periphery of the ellipse (for the case of the uniform sampling with the sampling period $t_{k+1}-t_{k}=0.75$ ) never leaves this ellipse and converges to the origin, while a trajectory starting not far from the ellipse remains outside the ellipse. 


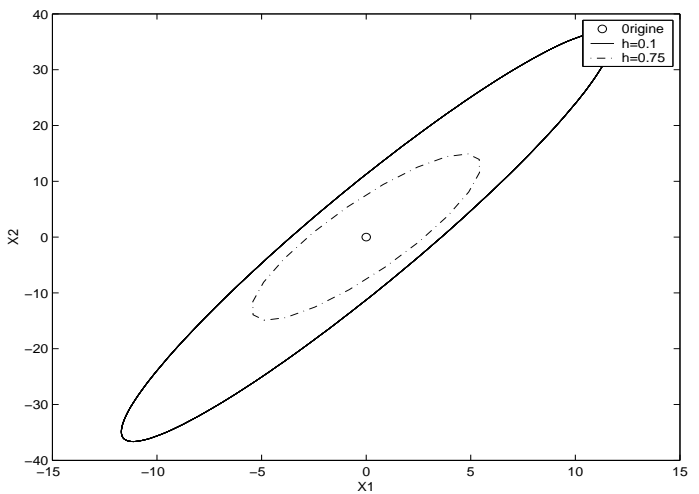

Figure 1: Ellipsoidal bounds on the domain of attraction

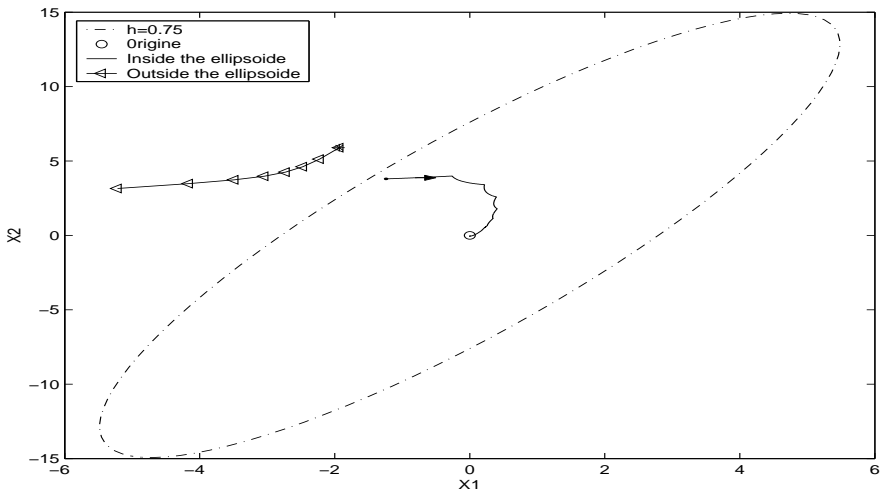

Figure 2: Stabilization result for $h=0.75$ 


\section{Conclusions}

A new approach to robust sampled-data stabilization of linear continuous-time systems is introduced. This approach is based on the continuous-time model with time-varying input delay. Under assumption that the maximum sampling interval is not greater than $h>0$, the $h$-dependent sufficient LMIs conditions for stabilization of systems with polytopic type uncertainty and for regional stabilization of systems with sampled-data saturated state-feedback are derived via descriptor system approach to time-delay systems.

The derived conditions are conservative since they guarantee stabilization for all sampling intervals not greater than $h$ and due to assumption (19). However, these conditions are simple. The problem of reducing their conservatism is currently under study. The new approach may be applied to a wide spectrum of robust sampled-data control problems.

\section{References}

Astrom, K. \& Wittenmark, B. (1989). Adaptive control. Reading, MA: Addison-Wesley.

Basar, T., \& Bernard, P. (1995) $H_{\infty}$ Optimal Control and Related Minimax Design Problems. A Dynamic Game Approach. Systems and Control: Foundation and Applications. Birkhauser, Boston.

Bamieh, B., Pearson, J., Francis, B. \& Tannenbaum, A. (1991) A lifting technique for linear periodic systems. Systems \& Control Letters, 17, 79-88.

Cao Y., Lin Z. \& Hu T.(2002). Stability analysis of linear time-delay systems subject to input saturation", IEEE Trans. Circuits and Systems, 49, 233-240.

Cao Y., Sun Y., \& Cheng, C. (1998). Delay-dependent robust stabilization of uncertain systems with multiple state delays. IEEE Trans. Automatic Control, 43, 1608-1612.

Chen, T., \& Francis, B. (1991). H2 optimal sampled-data control. IEEE Transactions on Automatic Control, 36, 387-397.

Dambrine, M., Richard, J.P. \& Borne, P. (1995). Feedback control of time-delay systems with bounded control and state. Mathematical Problems in Engineering, Overseas publishers association, 1, 77-87.

Dullerud, G. \& Glover, K. (1993) Robust stabilization of sampled-data systems to structured LTI perturbations. IEEE Trans. on Automat. Contr., 38, 1497-1508.

Fridman, E. (1992). Use of models with aftereffect in the problem of design of optimal digital control. (English. Russian original) Autom. Remote Control, 53, No. 10, 1523-1528.

Fridman, E. (2001). New Lyapunov-Krasovskii functionals for stability of linear retarded and neutral type systems. Systems 8 Control Letters, 43, 309-319.

Fridman, E. (2002). Stability of Linear Descriptor Systems with Delay: A Lyapunov-Based Approach. J. Math. Analy. Appl., 273, No 1, 24-44.

Fridman, E. \& Shaked, U. (2002). An improved stabilization method for linear time-delay systems. IEEE Trans. on Automat. Contr., 47, No. 11,1931-1937.

Fridman, E., Pila, A. \& Shaked, U. (2003). Regional stabilization and $H_{\infty}$ control of time-delay systems with saturating actuators. Int. J. Robust Nonlinear Control, 13, No.9, 885-907.

Gao, H. \& Wang, C. (2003). Comments and further results on a "Descriptor system approach to $H_{\infty}$ control of linear time-delay systems". IEEE Trans. on Automat. Contr., 48, No. 3, 520-525.

Hale, J. \& Lunel, S. (1993). Introduction to functional differential equations (New York: Springer-Verlag).

Hu, L., Cao, Y., Cheng, C. \& Shao, H. (2002). Sampled-data control for time-delay systems. J. of Franklin Institute, 339, 231-238.

Hu, L., Cao, Y. \& Shao, H. (2002). Constrained robust sampled-data control for nonlinear uncertain systems. Int. J. of Robust and Nonlinear Control, 12, 447-464.

Hu, L., Lam, J., Cao, Y., \& Shao, H. (2003). A LMI approach to robust $H_{2}$ sampled-data control for linear uncertain systems. IEEE Trans. Syst. Man Cybernetics, B, 33, 149-155.

Kolmanovskii, V. \& Myshkis, A. (1999). Applied Theory of functional differential equations, Kluwer.

Li, X. \& C. de Souza (1997). Criteria for robust stability and stabilization of uncertain linear systems with state delay. Automatica, 33, 1657-1662. 
Mikheev ,Yu.V., Sobolev V.A.\& Fridman E.M. (1988). Asymptotic analysis of digital control systems. (English. Russian original) Autom. Remote Control, 49, No. 9, 1175-1180.

Niculescu, S.I, de Souza, C.; Dugard, L., Dion, J.M. (1998) Robust exponential stability of uncertain systems with time-varying delays. IEEE Trans. Automat. Control, 43 , No. 5, 743-748.

Niculescu, S.I. (2001). Delay effects on stability: A Robust Control Approach, Lecture Notes in Control and Information Sciences, 269, Springer-Verlag, London, 2001.

Oishi, Y. (1997). A bound of conservativeness in sampled-data robust stabilization and its dependence on sampling periods. Systems 8 Control Letters, 32, 11-19.

Osborn, S. \& Bernstein, D. (1995). An exact treatment of the achievable closed-loop $\mathrm{H}_{2}$-performance of sampled-data controllers: From continuous-time to open-loop. Automatica 31, 4, 617-620.

Sivashankar, N. \& Khargonekar, P. (1994). Characterization of the $L_{2}$-induced norm for linear systems with jumps with applications to sampled-data systems, SIAM J. Control and Optimization, 32, 1128-1150.

Takaba, K., Morihira, N. \& Katayama, T. (1995). A generalized Lyapunov theorem for descriptor systems. Systems 83 Control Letters, 24, 49-51.

Tarbouriech S. \& Gomes da Silva, J. (2000). Synthesis of Controllers for Continuous-Time Delay Systems with Saturating Controls via LMI's. IEEE Trans. Automat. Contr., 45, No. 1, 105-111.

Trentelman, H. \& Stoorvogel, A. (1995). Sampled-data and discrete-time $H^{2}$ optimal control. SIAM J. Control and Optimization, 33, No.3, 834-862.

Yamamoto, Y. (1990). New approach to sampled-data control systems - a function space method, Proc. 29th Conf. on Decision and Control, Honolulu, HW, 1882-1887. 